\title{
SOLVING FOR THE PARAMETERS OF A BETA DISTRIBUTION UNDER TWO QUANTILE CONSTRAINTS
}

\author{
J. RENÉ VAN DORP* and THOMAS A. MAZZUCHI \\ Department of Engineering Management and Systems Engineering, \\ The George Washington University, Washington, DC 20052 USA
}

\begin{abstract}
It will be shown that a solution exists for the parameters of a beta distribution given any combination of a lower quantile and upper quantile constraint. A numerical procedure is developed to solve for the parameters of the beta distribution given these quantile constraints. Example solutions are provided.
\end{abstract}

\section{KEYWORDS}

Beta Parameters; Specified Quantiles; Numerical Procedure.

\section{INTRODUCTION}

Many authors (e.g. Gavaskar, 1988) have quoted the suitability of the beta distribution in different applications because of its flexibility. In classical analyses, estimation of the parameters of a beta distribution have concentrated on maximum likelihood estimation (Lau and Lau, 1991). In Bayesian analyses, methods for eliciting the parameters of a beta distributions have focused on eliciting: (a) a measure of central tendency such as the mean and a measure of dispersion such as the variance (e.g. Press, 1989, p. 40), (b) the mean and an additional quantile (e.g. Martz and Waller, 1982), or (c) equivalent observations (e.g. Gavaskar, 1988, Chaloner and Duncan, 1983, Cooke, 1991).

\footnotetext{
*Department of Engineering Management and Systems Engineering, 2130 H Street, Suite 704, Washington, DC 20052, E-mail: dorpjr@seas.gwu.edu.
} 
This paper will address the topic of solving for the parameters of a beta distribution given two distinct quantiles. Solving for the parameters of a beta distribution using two distinct quantiles involves using the incomplete beta function $B(x \mid a, b)$ given by

$$
B(x \mid a, b)=\frac{1}{\mathbb{B}(a, b)} \int_{0}^{x} u^{a-1}(1-u)^{b-1} d u
$$

where $a>0, b>0$ and $\mathbb{B}(a, b)=\frac{\Gamma(a) \cdot \Gamma(b)}{\Gamma(a+b)}$. The incomplete beta function $B(x \mid a, b)$ has no closed form. Weiler, 1965, resorts to solving for the parameters of the beta distribution given the $p$-th and $(1-p)$-th quantile graphically. This graphical approach, however, is limited to the number of graphs plotted. For intermediate solutions interpolation methods must be used, which may result in an interpolation error.

In this paper, the flexibility of the beta distribution will be reconfirmed by proving that a solution exists for the parameters of a beta distribution for any combination of a lower quantile and upper quantile constraint. Next, a numerical procedure will be described which solves for parameters of a beta distribution given a lower quantile and upper quantile constraint. The numerical procedure can be easily adapted to the case of Weiler, 1965, and improves the graphical method. In addition, the numerical procedure can be adapted to the case where the median and an additional quantile are specified as measures of central tendency and dispersion. In the next section, some properties of the beta distribution will be derived that are used to prove the theoretical result and to design the numerical procedure.

\section{SOME PROPERTIES OF THE BETA DISTRIBUTION}

Let $X \sim \operatorname{Beta}(\beta \cdot \alpha, \beta \cdot(1-\alpha))$, i.e.

$$
\operatorname{Pr}\{X \leq x \mid \alpha, \beta\}=\frac{1}{\mathbb{B}(\beta \cdot \alpha, \beta \cdot(1-\alpha))} \int_{0}^{x} u^{\beta \cdot \alpha-1}(1-u)^{\beta \cdot(1-\alpha)-1} d u
$$


where $0<\alpha<1, \beta>0$. Note that the chosen parameterization in (2) is different from the conventional parameterization in (1). Any beta distribution using the parameterization of (1) has a unique parameterization in (2) and vice versa. Using the parameterization of (2) it follows that

$$
E\left[X^{m} \mid \alpha, \beta\right]=\frac{\prod_{i=1}^{m}(\beta \cdot \alpha+m-i)}{\prod_{i=1}^{m}(\beta+m-i)}=\alpha \cdot \frac{\prod_{i=1}^{m-1}(\beta \cdot \alpha+m-i)}{\prod_{i=1}^{m-1}(\beta+m-i)}, m=1,2,3, \ldots
$$

with the convention that $\prod_{i=1}^{0}\{\cdot\}=1$. From $(3)$ it easily follows that

$$
\begin{gathered}
E[X \mid \alpha, \beta]=\alpha \\
\operatorname{Var}[X \mid \alpha, \beta]=\frac{\alpha \cdot(1-\alpha)}{(\beta+1)} .
\end{gathered}
$$

Consider the two different classes of degenerate distributions presented in Figure 1.

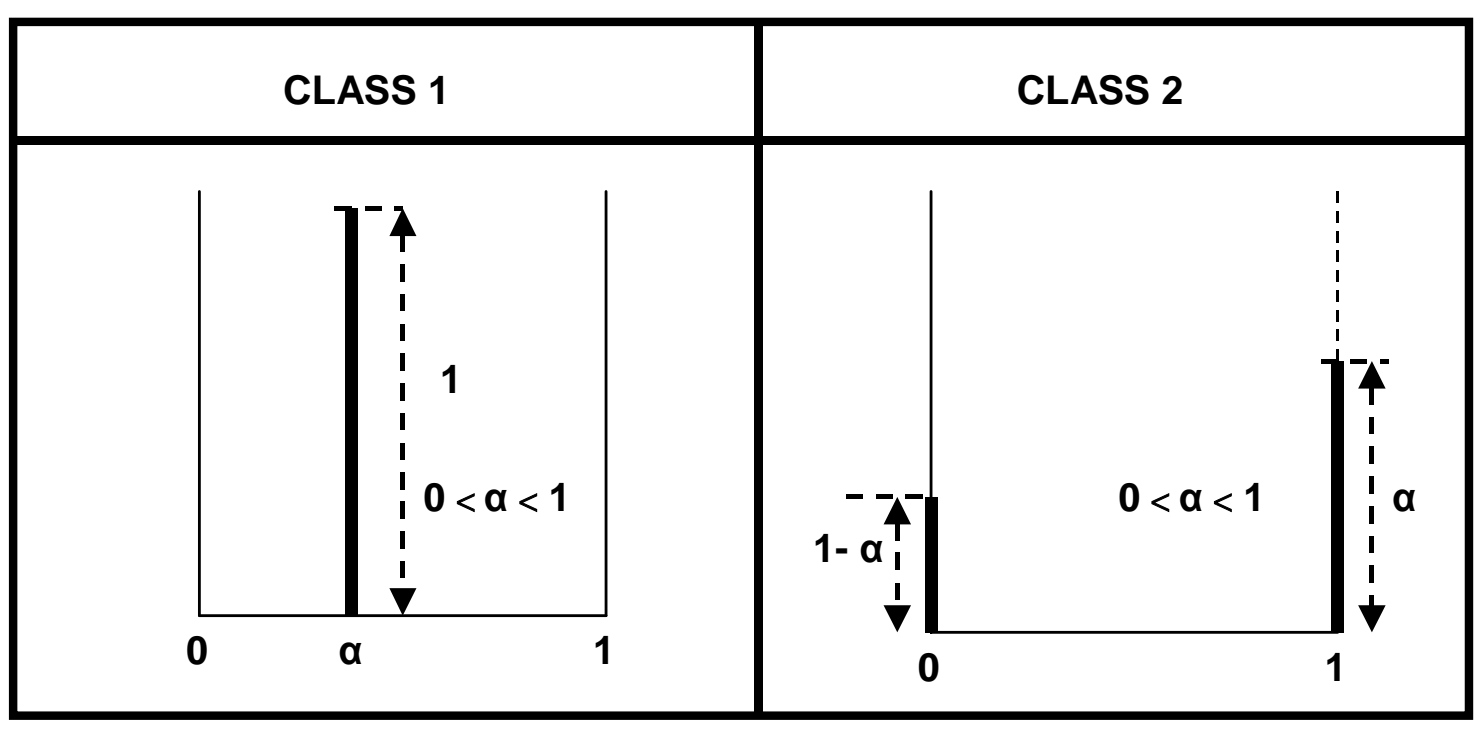

Figure 1. Two classes of degenerate beta distributions.

From (4) and (5) follows that the degenerate distribution in Class 1 of Figure 1 is the limiting distribution obtained by letting $\beta \rightarrow \infty$. With (3) follows that the moments of the limiting distribution by letting $\beta \downarrow 0$ coincide with the moments of the degenerate distribution in Class 2 
in Figure 1 (i.e. a Bernoulli with a point mass of $\alpha$ at 1). As both $X$ and the degenerate distribution of Class 2 have a bounded support, it follows that the degenerate distribution in Class 2 is the limiting distribution by letting $\beta \downarrow 0$ (see e.g. Harris, 1966, p. 103). Using the notation of (1), it has been shown (see e.g. Proschan and Singpurwalla, 1979) that for $b>0$

$$
0<a_{1}<a_{2} \Rightarrow B\left(x \mid a_{1}, b\right)>B\left(x \mid a_{2}, b\right), \forall \mathrm{x} \in(0,1),
$$

and for $a>0$

$$
b_{1}>b_{2}>0 \Rightarrow B\left(x \mid a, b_{1}\right)>B\left(x \mid a, b_{2}\right), \forall \mathrm{x} \in(0,1),
$$

From (6) and (7) it may be derived that

$$
\alpha_{2}>\alpha_{1}>0, \beta>0 \Rightarrow \operatorname{Pr}\left(X \leq x \mid \alpha_{2}, \beta\right)<\operatorname{Pr}\left(X \leq x \mid \alpha_{1}, \beta\right) .
$$

\section{THEORETICAL RESULT}

Definition 1 below introduces the quantile constraint concept used in the remainder of this paper.

Definition 1 : Let $0<x_{q}<1,0<q<1$. A random variable $X$ with support $[0,1]$ satisfies quantile constraint $\left(x_{q}, q\right) \Leftrightarrow \operatorname{Pr}\left\{X \leq x_{q}\right\}=q$.

As previously stated, the motivation of this paper is derived from the following problem..

Problem $\mathcal{P}:$ Solve $\alpha$ and $\beta$ for $X \sim \operatorname{Beta}(\beta \cdot \alpha, \beta \cdot(1-\alpha))$ under the two quantile constraints $\left(x_{q_{L}}, q_{L}\right)$ and $\left(x_{q_{U}}, q_{U}\right)$, where $q_{L}<q_{U}$.

Solving problem $\mathcal{P}$ involves the use of the incomplete beta function given by (1) and therefore has no closed form solution. Also, the quantile constraints in problem $\mathcal{P}$ can be considered a set of two nonlinear constraints in two unknowns, i.e. $\alpha$ and $\beta$, and as such does not necessarily have a feasible solution. To construct a numerical procedure with solves problem $\mathcal{P}$ 
in a finite number of iterations, it is necessary to prove that problem $\mathcal{P}$ has a solution for any combination of the two quantile constraints. This assertion will be proved in Theorem 1 through limiting arguments.

Theorem 1 : There exist a solution $\left(\alpha^{*}, \beta^{*}\right)$ to problem $\mathcal{P}$.

Proof : The proof involves four steps. In the first step it will be proved, using the notation in (2), that for a given $\beta>0$ and a quantile constraint $\left(x_{q}, q\right)$, a unique $\alpha^{\circ}$ exist such that $X \sim \operatorname{Beta}\left(\beta \cdot \alpha^{\circ}, \beta \cdot\left(1-\alpha^{\circ}\right)\right)$ satisfies that quantile constraint. In the second step it will be shown that for $\beta \downarrow 0$ the parameter $\alpha^{\circ} \rightarrow(1-q)$. In the third step it will be shown that for $\beta \rightarrow \infty$ the parameter $\alpha^{\circ} \rightarrow x_{q}$. Finally, in the fourth step, the statement of this theorem will be utilized.

Step 1 : Let a quantile constraint $\left(x_{q}, q\right)$ be specified for $X$. Assume that $\beta>0$ is given and introduce the function $\xi(\alpha, \beta)$ in $\alpha$ and $\beta$, where

$$
\xi(\alpha, \beta)=\operatorname{Pr}\left\{X \leq x_{q} \mid \alpha, \beta\right\}-q, 0<\alpha<1, \beta>0 .
$$

From the structure of $(2)$ it follows that $\xi(\alpha, \beta)$ is a continuous differentiable function for $0<\alpha<1, \beta>0$. Consider $\xi(\alpha, \beta)$ when $\alpha \downarrow 0$ and $\beta>0$ fixed. From (4) and (5) it follows, respectively, that

$$
\left\{\begin{array}{l}
\operatorname{Lim}_{\alpha \downarrow 0} E[X \mid \alpha, \beta]=0 \\
\operatorname{Lim}_{\alpha \downarrow 0} \operatorname{Var}[X \mid \alpha, \beta]=0
\end{array}\right.
$$

for any fixed $\beta>0$. Hence when $\alpha \downarrow 0$, the distribution of $X$ converges to a degenerate distribution with a single point mass at 0 . With $0<x_{q}<1,0<q<1$ it thus follows from (9) that 


$$
\operatorname{Lim}_{\alpha \downarrow 0} \xi(\alpha, \beta)=1-q>0
$$

for any fixed $\beta>0$. Similarly, using (4), (5) and using that the distribution of $X$ converges to a degenerate distribution with a single point mass at 1 when $\alpha \uparrow 1$, it follows that

$$
\operatorname{Lim}_{\alpha \uparrow 1} \xi(\alpha, \beta)=-q<0,
$$

for any fixed $\beta>0$. From (11), (12) and $\xi(\alpha, \beta)$ being a continuous function, it follows that

$$
\exists \alpha^{\circ} \in(0,1): \xi\left(\alpha^{\circ}, \beta\right)=0, \forall \beta>0 .
$$

Utilizing expression (8), it follows that $\xi(\alpha, \beta)$ is a strictly decreasing function in $\alpha$ for any fixed $\beta>0$. Thus, given fixed $\beta>0, \alpha^{\circ}$ is the unique solution to $\xi(\alpha, \beta)=0$ and $X \sim \operatorname{Beta}\left(\beta \cdot \alpha^{\circ}, \beta \cdot\left(1-\alpha^{\circ}\right)\right)$ satisfies the quantile constraint $\left(x_{q}, q\right)$ given fixed $\beta>0$. Before we proceed with Step 2, note that the solution $\alpha^{\circ}$ depends on $x_{q}$ (cf. (9)) and $\beta$ (cf. (13)) motivating the following notation

$$
\alpha^{\circ}=\mathcal{G}_{x_{q}}(\beta)
$$

where $\mathcal{G}_{x_{q}}(\cdot):(0, \infty) \rightarrow(0,1), 0<x_{q}<1,0<q<1$, such that

$$
\xi\left(\mathcal{G}_{x_{q}}(\beta), \beta\right)=0, \forall \beta>0 \text {. }
$$

From the structure of $(2),(15)$, and the implicit function theorem, it follows that $\mathcal{G}_{x_{q}}(\beta)$ is a continuous function for $\beta>0$. Using the definition of $\xi(\alpha, \beta)$ given by (9), and (15) it follows that

$$
\operatorname{Pr}\left\{X \leq x_{q} \mid \mathcal{G}_{x_{q}}(\beta), \beta\right\}=q, \forall \beta>0
$$

Step 2 : Consider $X \sim \operatorname{Beta}\left(\beta \cdot \alpha^{\circ}, \beta \cdot\left(1-\alpha^{\circ}\right)\right)$, where $\alpha^{\circ}=\mathcal{G}_{x_{q}}(\beta)$, and let $\beta \downarrow 0$. From continuity of $\operatorname{Pr}\left\{X \leq x_{q} \mid \mathcal{G}_{x_{q}}(\beta), \beta\right\}$ in $\beta$ for fixed $x_{q}$ it follows with (16) that

$$
\operatorname{Lim}_{\beta \downarrow 0} \operatorname{Pr}\left\{X \leq x_{q} \mid \mathcal{G}_{x_{q}}(\beta), \beta\right\}=q .
$$

From the structure of $(2)$ it has been shown that when $\beta \downarrow 0$, the distribution of $X$ converges to a 
degenerate distribution of Class 2, indicated in Figure 1. The limiting expectation of $X$ when $\beta \downarrow 0$ thus follows as the expectation of a Bernoulli random variable and from (17) it follows that

$$
\operatorname{Lim}_{\beta \downarrow 0} E\left[X \mid \mathcal{G}_{x_{q}}(\beta), \beta\right]=1-q .
$$

However, from (4) follows that

$$
E\left[X \mid \mathcal{G}_{x_{q}}(\beta), \beta\right]=\mathcal{G}_{x_{q}}(\beta),
$$

for any $\beta>0$ and it may be concluded with (18) and (19) that

$$
\operatorname{Lim}_{\beta \downarrow 0} \mathcal{G}_{x_{q}}(\beta)=1-q .
$$

Or in other words, when $\beta \downarrow 0$, the parameter $\alpha^{\circ} \rightarrow(1-q)$.

Step 3 : Consider $X \sim \operatorname{Beta}\left(\beta \cdot \alpha^{\circ}, \beta \cdot\left(1-\alpha^{\circ}\right)\right)$, where $\alpha^{\circ}=\mathcal{G}_{x_{q}}(\beta)$, and let $\beta \rightarrow \infty$. From (5) follows that when $\beta \rightarrow \infty$ the distribution of $X$ converges to a degenerate distribution of Class 1 in Figure 1 with a single point mass at some $x^{\bullet} \in[0,1]$.

From continuity of $\operatorname{Pr}\left\{X \leq x_{q} \mid \mathcal{G}_{x_{q}}(\beta), \beta\right\}$ in $\beta$ for fixed $x_{q}$ it follows from (16) that

$$
x^{\bullet}=x_{q}
$$

However, this means that,

$$
\operatorname{Lim}_{\beta \rightarrow \infty} E\left[X \mid \mathcal{G}_{x_{q}}(\beta), \beta\right]=x_{q} .
$$

Again from (4) it thus follows that

$$
\operatorname{Lim}_{\beta \rightarrow \infty} \mathcal{G}_{x_{q}}(\beta)=x_{q} .
$$

Or in other words, when $\beta \rightarrow \infty$, the parameter $\alpha^{\circ} \rightarrow x_{q}$.

Step 4 : Let $X \sim \operatorname{Beta}(\beta \cdot \alpha, \beta \cdot(1-\alpha))$. Let $\left(x_{q_{L}}, q_{L}\right)$ and $\left(x_{q_{U}}, q_{U}\right)$ be two quantile constraints specified for $X$, such that $q_{L}<q_{U}$. Consider the associated functions $\mathcal{G}_{x_{q_{L}}}(\beta)$ and $\mathcal{G}_{x_{q_{U}}}(\beta)$ each defined implicitly by $(9)$ and (15). Introducing the function

$$
H(\beta)=\mathcal{G}_{x_{q_{L}}}(\beta)-\mathcal{G}_{x_{q_{U}}}(\beta),
$$


it follows from (20) that

$$
\operatorname{Lim}_{\beta \downarrow 0} H(\beta)=\left(1-q_{L}\right)-\left(1-q_{U}\right)=q_{U}-q_{L}>0 .
$$

Similarly, from (23) it follows that

$$
\operatorname{Lim}_{\beta \rightarrow \infty} H(\beta)=x_{q_{L}}-x_{q_{U}}<0 .
$$

From the continuity of $\mathcal{G}_{x_{q_{L}}}(\beta)$ and $\mathcal{G}_{x_{q_{U}}}(\beta),(25)$ and (26) it follows that

$$
\exists \beta^{*}>0: H\left(\beta^{*}\right)=0 .
$$

Denoting $\alpha^{*}=\mathcal{G}_{x_{q_{L}}}\left(\beta^{*}\right)$ (cf. (14)), it follows from (27) that

$$
\alpha^{*}=\mathcal{G}_{x_{q_{L}}}\left(\beta^{*}\right)=\mathcal{G}_{x_{q_{U}}}\left(\beta^{*}\right) .
$$

In other words, $X \sim \operatorname{Beta}\left(\beta^{*} \cdot \alpha^{*}, \beta^{*} \cdot\left(1-\alpha^{*}\right)\right)$ satisfies both quantile constraints $\left(x_{q_{L}}, q_{L}\right)$ and $\left(x_{q_{U}}, q_{U}\right)$ and thus $\left(\alpha^{*}, \beta^{*}\right)$ is a solution to problem $\mathcal{P}$.

Theorem 1 proves the existence of a solution to problem $\mathcal{P}$. The uniqueness of the solution $\left(\alpha^{*}, \beta^{*}\right)$ to $\mathcal{P}$ would follow by showing that; (1) $H(\beta)$ has 0 or 1 stationary points for $\beta>0$; (2) if $H(\beta)$ has a stationary point for $\beta>0$ this stationary point coincides with a global maximum. It is conjectured that the above assertion holds. Numerical analyses in the examples below support this conjecture. In case multiple solutions exist to problem $\mathcal{P}$, the numerical algorithm below is designed so that the selected solution coincides with the solution with the lowest value for $\beta^{*}$, and thus the highest level of uncertainty. The later solution would be a preferred solution in case $x_{q_{L}}$ and $x_{q_{U}}$ are elicited through expert judgment.

\section{DESIGN OF A NUMERICAL PROCEDURE}

As problem $\mathcal{P}$ cannot be solved in closed form, a numerical procedure that finds a solution to problem $\mathcal{P}$ with a prescribed level of accuracy in a finite number of iterations, is desirable. Below, such a numerical procedure will be described informally. The numerical 
method uses a procedure to solve for the $q$-th quantile of a beta distribution with known parameters. Such a procedure is described in the appendix.

With (4) and (5) follows that $\alpha$ is a location parameter and $\beta$ is an uncertainty parameter given the value of $\alpha$, where higher values of $\beta$ coincide with lower uncertainty levels. These interpretations of the parameters $\alpha$ and $\beta$ are used in the design of the numerical procedure to find a solution to $\mathcal{P}$. Assume for now that an interval $\left[a_{1}, b_{1}\right]$ is established containing $\beta^{*}$ which yields a solution $\left(\alpha^{*}, \beta^{*}\right)$ of $\mathcal{P}$, where $\alpha^{*}=\mathcal{G}_{x_{q}}\left(\beta^{*}\right)$. Let $\beta_{1}$ be the midpoint of this interval. The $k$-th iteration of the numerical procedure is described below.

To solve $\left(\alpha^{\circ}\right)_{k}$ satisfying the quantile constraint $\left(x_{q_{U}}, q_{U}\right)$ of $\mathcal{P}$ given a value for $\beta_{k}$, successive shrinking intervals $\left[d_{n}, e_{n}\right]$ will be calculated containing the solution $\left(\alpha^{\circ}\right)_{k}$. With $(2)$ follows that $\left(\alpha^{\circ}\right)_{k} \in[0,1]$. Hence, $\left[d_{1}, e_{1}\right]=[0,1]$. Next, $\alpha_{n}$ is set to the midpoint of $\left[d_{n}, e_{n}\right]$ after which the probability mass $\left(q_{U}\right)_{n}=\operatorname{Pr}\left\{X \leq x_{q_{U}} \mid \alpha_{n}, \beta_{k}\right\}$ is calculated. In case $\left(q_{U}\right)_{n} \leq q_{U}$, the beta distribution is skewed too much towards 1. Therefore, with (4) it follows that the value of the location parameter $\alpha_{n}$ is too high. Hence, the next interval containing $\left(\alpha^{\circ}\right)_{k}$ is $\left[d_{n+1}, e_{n+1}\right]=\left[d_{n}, \alpha_{n}\right]$. Vice versa, in case $\left(q_{U}\right)_{n}>q_{U}$, the beta distribution is skewed too much towards 0 . Therefore, with (4) it follows that the value of the location parameter $\alpha_{n}$ is too small. Hence, the next interval which contains $\left(\alpha^{\circ}\right)_{k}$ can be set to $\left[d_{n+1}, e_{n+1}\right]=\left[\alpha_{n}, e_{n}\right]$. Finally, the next estimate $\alpha_{n+1}$ is set to be the midpoint of the interval $\left[d_{n+1}, e_{n+1}\right]$. The above procedure is repeated until $\left(q_{U}\right)_{n}$ is close to $q_{U}$ with a desired level of accuracy. The quantile constraint $\left(x_{q_{U}}, q_{U}\right)$ of $\mathcal{P}$ is met once this accuracy has been reached and $\left(\alpha^{\circ}\right)_{k}$ is set equal to the last generated $\alpha_{n}$.

After solving for $\left(\alpha^{\circ}\right)_{k}$, the procedure calculates the $q_{L}$-th quantile $\left(x_{q_{L}}\right)_{k}$ of $\operatorname{Beta}\left(\beta_{k} \cdot\left(\alpha^{\circ}\right)_{k}, \beta_{k} \cdot\left(1-\left(\alpha^{\circ}\right)_{k}\right)\right)$. In case $\left(x_{q_{L}}\right)_{k}<x_{q_{L}}$ the uncertainty in $\operatorname{Beta}\left(\beta_{k} \cdot\left(\alpha^{\circ}\right)_{k}\right.$, $\left.\beta_{k} \cdot\left(1-\left(\alpha^{\circ}\right)_{k}\right)\right)$ is too high. Therefore, the current estimate of the uncertainty parameter $\beta_{k}$ is too low. Hence, the next interval which contains $\beta^{*}$ can be set to $\left[a_{k+1}, b_{k+1}\right]=\left[\beta_{k}, b_{k}\right]$. Vice versa, if $\left(x_{q_{L}}\right)_{k}>x_{q_{L}}$ the uncertainty in $\operatorname{Beta}\left(\beta_{k} \cdot\left(\alpha^{\circ}\right)_{k}, \beta_{k} \cdot\left(1-\left(\alpha^{\circ}\right)_{k}\right)\right)$ is too low. Therefore, the current estimate of the uncertainty parameter $\beta_{k}$ is too high. Hence, the next interval which 
contains $\beta^{*}$ can be set to $\left[a_{k+1}, b_{k+1}\right]=\left[a_{k}, \beta_{k}\right]$. Finally, the next estimate $\beta_{k+1}$ is taken to be the midpoint of the interval $\left[a_{k+1}, b_{k+1}\right]$. The above procedure is repeated until the current estimate $\left(x_{q_{L}}\right)_{k}$ is close to $x_{q_{L}}$ with a desired level of accuracy. The quantile constraint $\left(x_{q_{L}}, q_{L}\right)$ of $\mathcal{P}$ is met once this accuracy has been reached. The parameters $\left(\alpha^{*}, \beta^{*}\right)$ solving $\mathcal{P}$ are set equal to the last generated tuple $\left(\left(\alpha^{\circ}\right)_{k}, \beta_{k}\right)$.

To establish a starting interval $\left[a_{1}, b_{1}\right]$ containing $\beta^{*}$ the following steps may be adopted in the procedure. Set the lower bound $a_{1}=0$. To obtain the upper bound $b_{1}$, set $\beta_{1, k}=1$, where $k=1$, and solve for $\left(\alpha^{\circ}\right)_{1, k}$ satisfying the quantile constraint $x_{q_{U}}$ of problem $\mathcal{P}$ as described in the previous paragraph. Next, solve for the $q_{L}$-th quantile $\left(x_{q_{L}}\right)_{1, k}$ of $\operatorname{Beta}\left(\beta_{1, k} \cdot\left(\alpha^{\circ}\right)\right.$ $\left.{ }_{1, k}, \beta_{1, k} \cdot\left(1-\left(\alpha^{\circ}\right)_{1, k}\right)\right)$. In case $\left(x_{q_{L}}\right)_{1, k}<x_{q_{L}}$ the uncertainty in $\operatorname{Beta}\left(\beta_{1, k} \cdot\left(\alpha^{\circ}\right)\right.$ $\left.{ }_{1, k}, \beta_{1, k} \cdot\left(1-\left(\alpha^{\circ}\right)_{1, k}\right)\right)$ is too high. Therefore, $\beta_{1, k}<\beta^{*}$. In that case, set $\beta_{1, k+1}=2 \cdot \beta_{1, k}$ and repeat the above procedure. Vice Versa, in case $\left(x_{q_{L}}\right)_{1, k}>x_{q_{L}}$ the uncertainty in $\operatorname{Beta}\left(\beta_{1, k} \cdot\left(\alpha^{\circ}\right)_{1, k}, \beta_{1, k} \cdot\left(1-\left(\alpha^{\circ}\right)_{1, k}\right)\right)$ is too low. Therefore, $\beta_{1, k}>\beta^{*}$. In that case, set $b_{1}=\beta_{1, k}$ and the starting interval $\left[a_{1}, b_{1}\right]$ has been established. Note that, in case multiple solutions exist to problem $\mathcal{P}$, the starting interval is chosen such that the selected solution to $\mathcal{P}$ by the algorithm coincides with the solution with the lowest value for $\beta^{*}$, and thus the highest level of uncertainty.

The numerical procedure described in this section can be summarized by three different bisection methods (See for example Press et al., 1989) BISECT 1, BISECT2 and BISECT 3 and are described in the Appendix in Pseudo Pascal.

\section{EXAMPLES}

The authors implemented the bisection methods described in the previous section in a PC-based program BETA-CALCULATOR. The accuracy for $\delta$ in the bisection methods BISECT1 and BISECT2 was set to $10^{-8}$. The accuracy in the bisection method BISECT3 was set to $10^{-4}$. Table I contains solutions to problem $\mathcal{P}$ for 5 different combinations of a lower quantile and upper quantile constraint calculated using BETA-CALCULATOR. In addition, 
Table I provides the maximum number of iterations in each bisection method to yield the solutions with the above settings of error tolerances.

Table I. Some Calculation Examples

\begin{tabular}{l|l|l|l|l|l|l|l|l}
\hline & & $q$ & $x_{q}$ & $\alpha^{*}$ & $\beta^{*}$ & $\# 1$ & $\# 2$ & $\# 3$ \\
\hline Example 1 & $L$ & 0.05 & 0.15 & 0.886 & 0.540 & 23 & 25 & 12 \\
& $U$ & 0.15 & 0.75 & & & & & \\
\hline Example 2 & $L$ & 0.65 & 0.65 & 0.615 & 32.000 & 28 & 26 & 1 \\
& $U$ & 0.95 & 0.75 & & & & & \\
\hline Example 3 & $L$ & 0.49 & 0.25 & 0.267 & 11.390 & 71 & 24 & 11 \\
& $U$ & 0.99 & 0.60 & & & & & \\
\hline Example 4 & $L$ & 0.25 & 0.25 & 0.338 & 14.375 & 45 & 24 & 8 \\
& $U$ & 0.95 & 0.55 & & & & & \\
\hline Example 5 & $L$ & 0.05 & 0.45 & 0.633 & 19.562 & 44 & 25 & 10 \\
& $U$ & 0.95 & 0.80 & & & & & \\
\hline
\end{tabular}

The figures below have been generated using BETA-CALCULATOR. Figure 2 and

Figure 3 contain examples of the implicit functions $G_{x_{q_{L}}}(\beta)$ and $G_{x_{q_{U}}}(\beta)$ associated with Example 1 and Example 5, respectively. Finally, Figure 4 contains plots of the function $H(\beta)$ for the examples in Table I. It may be observed from the examples in Figure 4 that: (a) $H(\beta)$ has 0 or 1 stationary points for $\beta>0$ and (b) if $H(\beta)$ has a stationary point for $\beta>0$ this point coincides with a global maximum. The analysis in Figure 4 supports the conjecture in Section 3 , i.e. that the solution of the parameters of a beta distribution subject to a lower quantile constraint and upper quantile constraint exists and is unique.

\section{ACKNOWLEDGMENT}

The authors would like to acknowledge R.M. Cooke for providing motivating comments to develop this paper. We extend our gratitude to S. Kotz for his valuable comments in structuring the proof of Theorem 1 and to the Associate Editor and the referee for their most helpfull comments. 


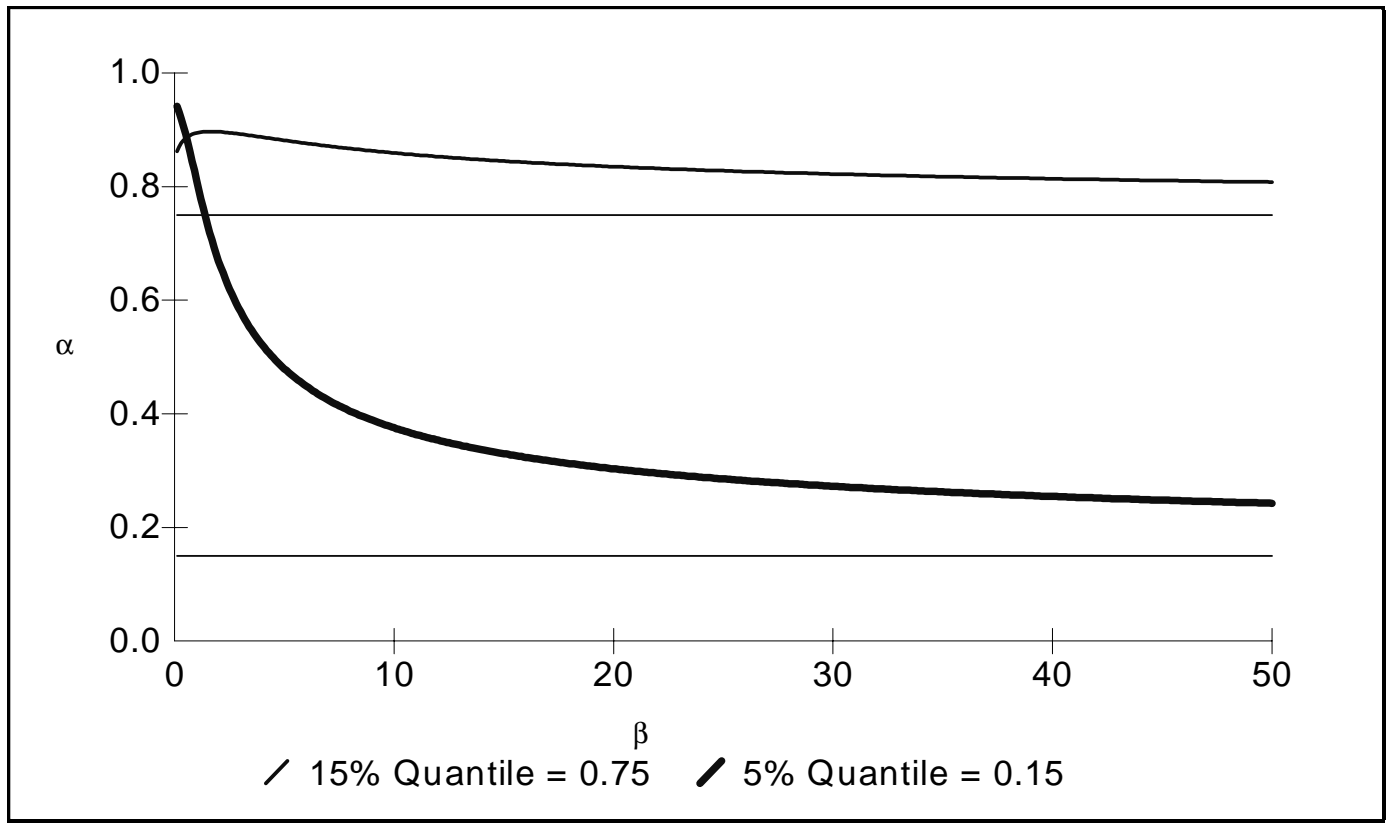

Figure 2. A plot of $G_{x_{q_{L}}}(\beta)$ and $G_{x_{q_{U}}}(\beta)$ for Example 1 in Table I.

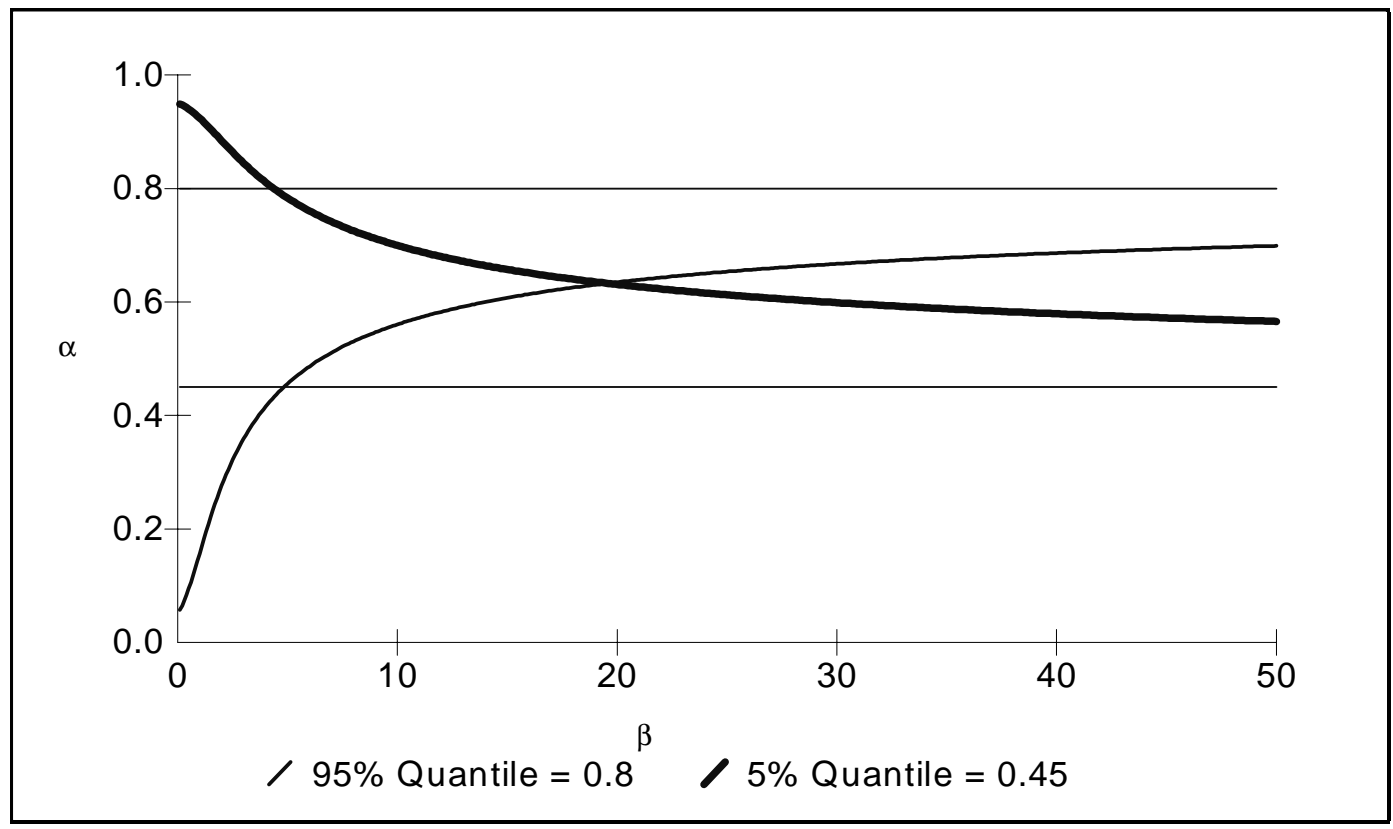

Figure 3. A plot of $G_{x_{q_{L}}}(\beta)$ and $G_{x_{q_{U}}}(\beta)$ for Example 5 in Table I. 


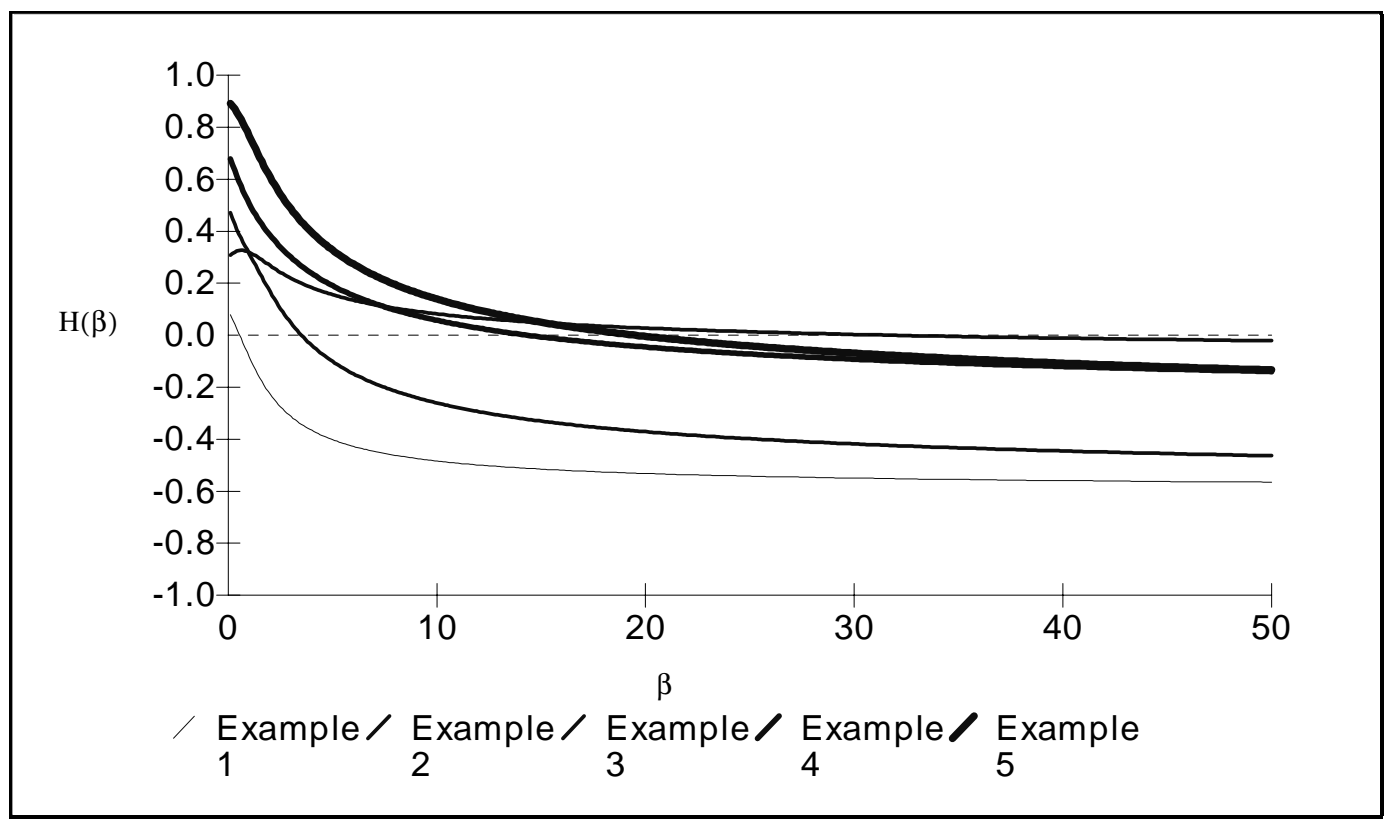

Figure 4. Plot of the function $H(\beta)$ for the Examples in Table I.

\section{REFERENCES}

Chaloner, K.M and Duncan, G.T. (1983). Assessment of a Beta Prior Distribution: PM Elicitation. The Statistician, 32, 174 - 181.

Cooke, R.M. (1991). Experts in Uncertainty: Opinions and Subjecive Probability in Science. Oxford University Press, Oxford.

Gavaskar, U. (1988). A Comparison of Two Elicitation Methods for a Prior Distribution for a Binomial Parameter. Management Science, 34, 784 - 790.

Harris, B. (1966). Theory of Probability. Addision-Wesley, Reading.

Lau, H. and Lau, A. (1991). Effective Procedures for Estimating Beta Distribution's Parameters and their Confidence Intervals. Journal of Statistical Computation and Simulation, 38, 139-150.

Martz, H.F. and Waller, R.A. (1982). Bayesian Reliability Analysis. Wiley, New York.

Press, S.J. (1989). Bayesian Statistics, Principles, Models and Applications. Wiley, New York. 
Press, W.H., Flannery, B.P., Teukolsky S.A. and Vettering W.T. (1989). Numerical Recipes in Pascal, Cambridge University Press, Cambridge.

Proschan, F. and Singpurwalla, N.D. (1979). Accelerated Life Testing - A Pragmatic Bayesian Approach. In: J. Rustagi, Optimizing Methods in Statistics, Academic Press, New York, 385 401.

Weiler, H. (1965). The Use of Incomplete Beta Functions for Prior Distributions in Binomial Sampling. Technometrics, 7, 335 - 346.

\section{APPENDIX}

Let $X \sim \operatorname{Beta}(\beta \cdot \alpha, \beta \cdot(1-\alpha))$. The bisection methods below use a numerical algorithm given in Press et al. (1989) to evaluate the incomplete beta function $B(\cdot \mid a, b)$ given by (1). $\operatorname{BISECT1}(\alpha, \beta, q)$ solves for the q-th quantile $x_{q}$ of $X . B I S E C T 2\left(x_{q}, \beta, q\right)$ solves for the parameter $\alpha^{\circ}$ satisfying the quantile constraint $\left(x_{q}, q\right)$. BISECT3 $\left(x_{q_{L}}, x_{q_{U}}, q_{L}, q_{U}\right)$ solve for a solution $\left(\alpha^{*}, \beta^{*}\right)$ solving $P$. A method to determine a starting interval $\left[a_{1}, b_{1}\right]$ containing $\beta^{*}$ is given in STEP 1, STEP 2, and STEP 3 of BISECT 3.

$$
\begin{array}{ll}
\boldsymbol{B I S E C T}(\boldsymbol{\alpha}, \boldsymbol{\beta}, \boldsymbol{q}): \\
\text { STEP } 1 & m:=1 ; \text { Set }\left[d_{1}, e_{1}\right]=[0,1] ; \\
\text { STEP2 } & x_{q, m}:=\frac{d_{m}+e_{m}}{2} ; q_{m}:=B\left(x_{q, m} \mid \beta \cdot \alpha, \beta \cdot(1-\alpha)\right) ; \\
\text { STEP3 } & \text { If } q_{m} \leq q \text { then } d_{m+1}:=x_{q, m} ; e_{m+1}:=e_{m} ; \\
& \text { Else } e_{m+1}:=x_{q, m} ; d_{m+1}:=d_{m} ; \\
\text { STEP 4 } & \text { If }\left|q_{m}-q\right|<\delta \text { then Stop; } \\
& \text { Else } m:=m+1 ; \text { GotoSTEP } 2 ;
\end{array}
$$

$B I S E C T 2\left(x_{q}, \beta, q\right):$

STEP $1 \quad n:=1 ; \operatorname{Set}\left[d_{1}, e_{1}\right]=[0,1] ;$ 
STEP $2 \quad \alpha_{n+1}:=\frac{d_{n}+e_{n}}{2} ; q_{n}:=B\left(x_{q} \mid \beta \cdot \alpha_{n+1}, \beta \cdot\left(1-\alpha_{n+1}\right)\right)$;

STEP3 If $q_{n} \leq q$ then $e_{n+1}:=\alpha_{n+1} ; d_{n+1}:=d_{n}$;

Else $d_{n+1}=\alpha_{n+1} ; e_{n+1}:=e_{n}$;

STEP $4 \quad$ If $\left|q_{n}-q\right|<\delta$ then Stop;

Else $n:=n+1 ;$ Goto STEP 2 ;

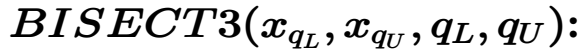

STEP $1 \quad k:=1 ; \beta_{1, k}:=1$;

$\operatorname{STEP} 2\left(\alpha^{\circ}\right)_{1, k}:=\operatorname{BISECT2}\left(x_{q_{U}}, \beta_{1, k}, q_{U}\right)$;

$\left(x_{q_{L}}\right)_{1, k}:=\operatorname{BISECT1}\left(\left(\alpha^{\circ}\right)_{1, k}, \beta_{1, k}, q_{L}\right)$;

STEP3 If $\left(x_{q_{L}}\right)_{1, k}<x_{q_{L}}$ then $\beta_{1, k+1}:=2 \cdot \beta_{1, k} ;$ Goto STEP 2 ;

Else $\left[a_{1}, b_{1}\right]:=\left[0, \beta_{1, k}\right]$;

STEP $4 \quad k:=1 ; \beta_{1}:=\frac{a_{1}+b_{1}}{2}$;

$\operatorname{STEP} 5\left(\alpha^{\circ}\right)_{k}:=\operatorname{BISECT} 2\left(x_{q_{U}}, \beta_{k}, q_{U}\right) ;\left(x_{q_{L}}\right)_{k}:=\operatorname{BISECT} 1\left(\left(\alpha^{\circ}\right)_{k}, \beta_{k}, q_{L}\right)$;

STEP6 If $\left(x_{q_{L}}\right)_{k}<x_{q_{L}}$ then $a_{k+1}:=\beta_{k} ; b_{k+1}:=b_{k}$;

Else $a_{k+1}:=a_{k} ; b_{k+1}:=\beta_{k}$

STEP7 If $\left|\left(x_{q_{L}}\right)_{k}-x_{q_{L}}\right|<\delta$ then Stop

Else $k:=k+1 ;$ Goto STEP 5 ; 